\title{
FORCE-DEFLECTION BEHAVIOR OF A SMART ATTACHMENT MECHANISM
}

\author{
Joseph W. Clement, AIAA Member, Graduate Student \\ Diann E. Brei, AIAA Member, Associate Professor \\ Department of Mechanical Engineering \\ The University of Michigan \\ 2250 G.G. Brown, Ann Arbor MI 48109-2125
}

\begin{abstract}
Active Velcro is a unique form of distributed actuation that generates relative planar motion between two surfaces while maintaining a positive connection. The key distinguishing feature of this active surface is its ability to engage and retain the guest surface. This paper focuses on understanding the mechanism behind this ability. An analytical model that captures the quasi-static force-deflection behavior of this complex mechanism under general loading conditions is derived, while specific models for engagement and retention are presented. The validity of the models was experimentally verified under three common operating scenarios: free deflection, surface positioning and guest surface engagement/retention. The impact of manufacturing effects, friction and plastic deformation are examined. Significant improvement over MEMs based active surfaces is demonstrated.
\end{abstract}

\section{INTRODUCTION}

A new paradigm in actuation technologies, distributed active surfaces, is very useful for moving objects long distances and generating complex motions. There have been a variety of active surfaces developed at the microscale $^{1-9}$ and macroscale ${ }^{10,11}$ for applications as varied as automated part sorting ${ }^{8}$, biologic tissue conveyance $^{11}$, parcel manipulation ${ }^{10}$, and fiber optic coupling. A unique active surface, nicknamed Active Velcro, offers both planar translation and rotation capabilities while providing a secure retention force between the surfaces. Active Velcro has two surfaces: 1) a host, comprised of a highly redundant array of active prongs and 2) a guest, comprised solely of a sparsely patterned array of inactive posts (Figure 1a). The two surfaces snap-fit together, similar to Velcro, when brought into contact by entrapping the post within a group of three prongs, termed a cell. The cell is activated by electrically heating an SMA wire that is connected to the prongs via suspension lines attached to an actuation triangle (Figure 1b). When the wire pulls down the actuation triangle, the cell closes in a grasping action.
The grasping action created by heating the SMA wire can be employed to increase the hold on a guest surface but by itself is insufficient to create relative motion between the two surfaces. However, the interconnectivity of cells within the host surface can be exploited to create the more complex prong motions necessary to move the guest surface. When two adjacent SMA wires are activated, the adjacent cells will attempt to close with the same grasping action, but the prong shared by both cells $\left(\mathrm{B}_{1}=\mathrm{C}_{2}\right)$ will move according to the resultant force vector which is oriented perpendicular to the line connecting the two energized SMA wires (Figure 2). This prong $\left(\mathrm{B}_{1}\right)$ pushes the post to the next cell and the process can be repeated resulting in a saw-toothed translation across the host surface. Utilizing guest surfaces with multiple posts, the positioning forces can be increased linearly while providing redundancy to improve reliability. By altering the timing and directions of positioning forces applied to each guest surface post, rotation can also be achieved. Experimental testing of prototype Active Velcro surfaces has successfully demonstrated full planar motion at translational speeds up to $2.8 \mathrm{~mm} / \mathrm{s}$ and rotational speeds up to $2.5 \mathrm{deg} / \mathrm{s}$ for multi-post guest surfaces ${ }^{12}$.

The distinguishing feature of Active Velcro is its ability to maintain a positive connection between the two surfaces; all other active surfaces ${ }^{1-11}$ rely on gravity assistance to maintain engagement. Active Velcro was designed so that the connection forces (engagement, retention) and required forces for positioning (translation, rotation) can be tailored by altering key features of the connection topology depicted in Figure 3. The post and prong lead surfaces determine the engagement force. For example, elongating the lead surface of the prongs from a spherical to a conical shape reduces the engagement force without affecting either the retention or positioning forces. The trailing surfaces of both the post and prong determine the retention force. Increasing the slope of the prong's trailing surface reduces the retention force without affecting engagement or positioning forces. The 


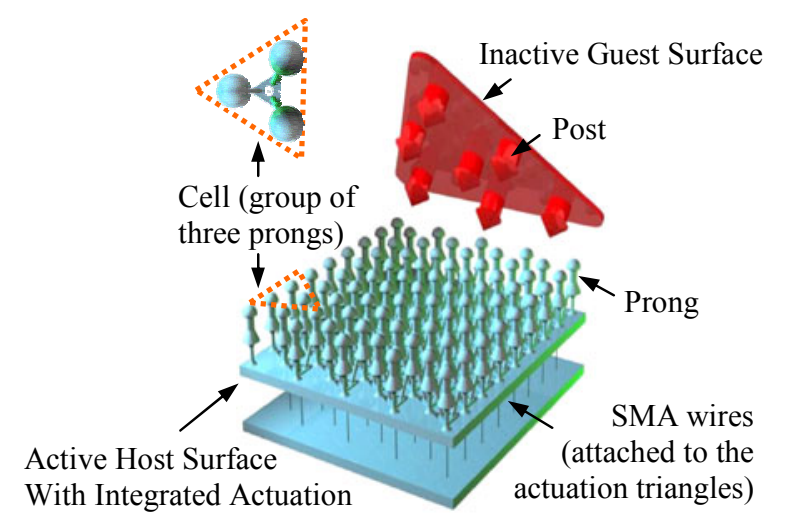

a) Active Velcro System

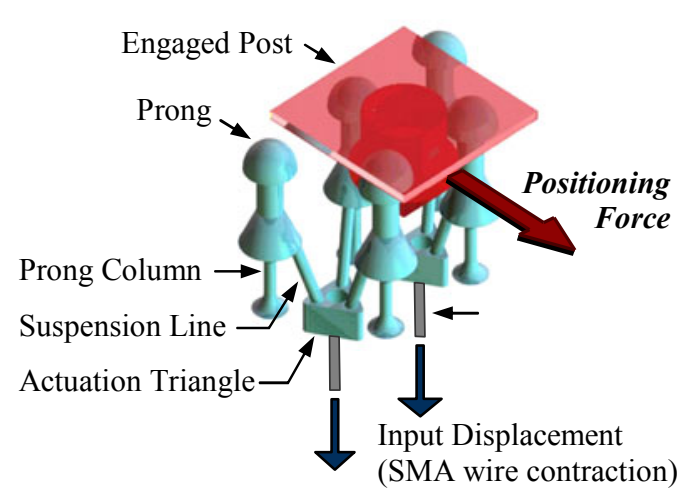

b) Close-Up (Three Cells Shown)

Figure 1: Active Velcro System Overview. a) Active Velcro consists of two surfaces: one inactive guest surface with a sparse arrangement of posts and an active host surface with a densely distributed array of actuated prongs. The host surface contains all the actuation, power and control systems and when activated inches the guest surface to the desired position and orientation. b) The prongs are activated by straining SMA wires located at the center of alternating groups of three prongs (termed a cell). When a given SMA is energized, the deflection of the SMA wire acts on the center of the actuation triangle, which in turn distributes the work to the three attached prongs via three suspension lines.

support columns and diameters of the connecting heads of both the post and prong primarily impact the positioning force. Decreasing the diameter of the support column for the guest surface post with respect to a given prong head diameter increases the positioning force by allowing the post to easily slip through the channels in the host surface array, thereby reducing any interference to motion.

The snap-fit connection is the fundamental characteristic that enables Active Velcro to manipulate objects in unstable moving frames or the low gravity environment of space. Therefore, it is critical that an understanding of the complex interactions between the

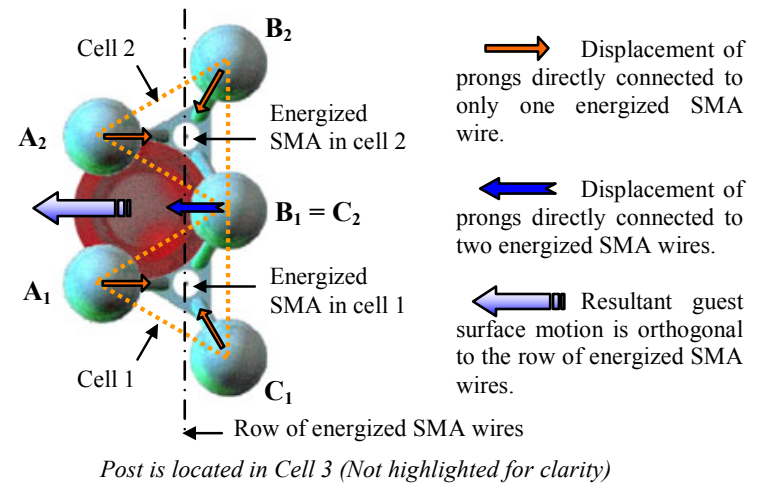

Figure 2: Local Prong Operation. If the post is located in a cell without an SMA wire it can be translated to the left of the figure by energizing two SMA wires. When the two SMA wires are energized prongs $A_{1}, C_{1}, A_{2}$, and $B_{2}$ will deflect towards their respective attached and energized SMA wires. Both SMA wires act upon prong $B_{1}$; therefore its deflection is given by the resultant force vector created by the energized wire. As a result of the individual prong motions, prong $B_{1}$ pushes the post between prongs $A_{1}$ and $A_{2}$ to complete the translation. A similar process can be employed when the post is located in a cell containing an SMA wire only it requires the activation of three SMA wires to generate guest surface motion. host and guest surfaces are accurately captured so that an engineer can predict the forces required to engage the guest and host surfaces, the strength of the snap-fit connection, and the positioning forces offered by the technology. In this paper, quasi-static force-deflection models are derived and experimentally validated under three scenarios representative of common operational conditions: free deflection, positioning, and engagement/retention.

\section{FORCE-DEFLECTION MODEL FORMULATION}

Key to capturing the general quasi-static behavior (engagement, retention, positioning force) for the entire host surface is understanding how the prongs and actuation triangle within an individual cell move under applied loads. An individual cell is modeled with two types of elements: 1) flexible elements (prong column and suspension line) denoted by subscript $i$, that store strain energy and 2) rigid elements (actuation triangle and all other elements within the prong) that provide connectivity for the transmission of forces and displacements. The nomenclature for these elements is

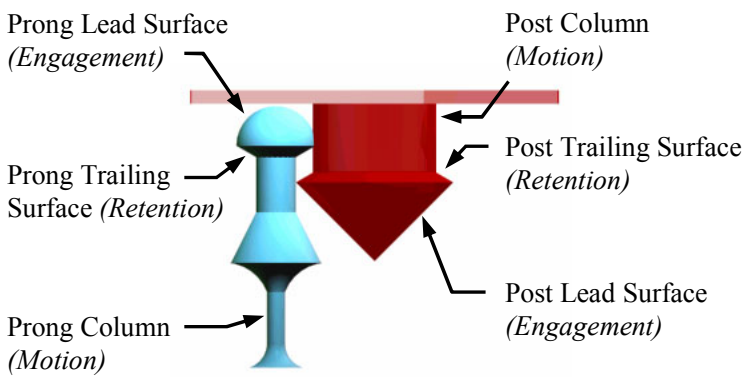

Figure 3: Connection Topologies. The surfaces most directly affecting engagement, retention, translation, and rotation can be independently tailored. 


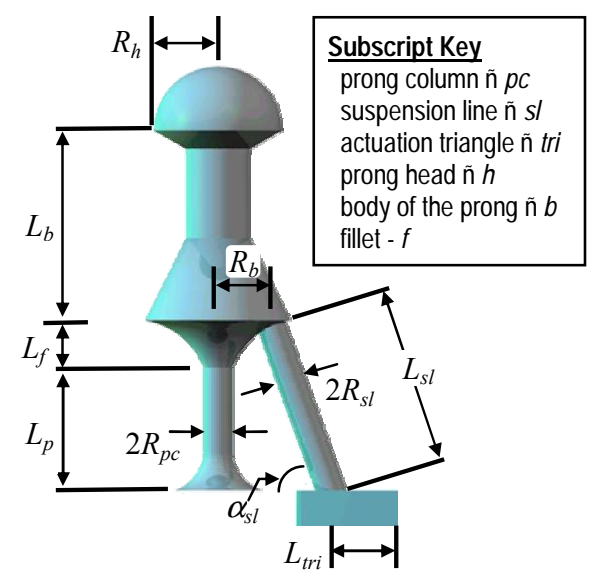

Figure 4: Variable Dimensional Parameters. Ten parameters are required to fully define the host surface geometry. For the modeling effort, only the prong column and suspension line were considered flexible elements (geometry denoted by the subscripts $p c$ and $s /$ respectively).

shown for a single prong in Figure 4. For this analysis it was assumed that all these elements are made from the same material and do not experience buckling. The SMA wire was modeled as a simple force input and does not add stiffness to the structure.

If damping is ignored the host surface can be modeled as a system stiffness matrix, $\boldsymbol{K}_{n e t}$, that relates the vector of externally applied loads, $\boldsymbol{F}$, to the global displacements, $\boldsymbol{D}$, (Figure 5):

$$
\left\{\begin{array}{lllll}
F_{1} & \cdots & F_{j} & \cdots & F_{s}
\end{array}\right\}^{T}=K_{n e t}\left\{\begin{array}{lllll}
D_{1} & \cdots & D_{j} & \cdots & D_{s}
\end{array}\right\}^{T} .
$$

Generally the prongs and actuation triangles are able to move with six degrees of freedom, thus $\boldsymbol{F}_{\boldsymbol{j}}$ and $\boldsymbol{D}_{\boldsymbol{j}}$ are actually $6 \times 1$ vectors:

$$
\boldsymbol{F}_{\boldsymbol{j}}=\left\{\begin{array}{llllll}
\boldsymbol{F}_{\boldsymbol{j}_{z}} & \boldsymbol{M}_{\boldsymbol{j}_{\varphi_{z}}} & \boldsymbol{F}_{\boldsymbol{j}_{x}} & \boldsymbol{M}_{\boldsymbol{j}_{\psi_{y}}} & \boldsymbol{F}_{\boldsymbol{j}} & \boldsymbol{M}_{\boldsymbol{j}_{\psi_{x}}}
\end{array}\right\}^{\boldsymbol{T}},
$$

$$
\boldsymbol{D}_{\boldsymbol{j}}=\left\{\begin{array}{llllll}
\Delta_{\boldsymbol{j}_{z}} & \Delta_{\boldsymbol{j}_{\varphi_{z}}} & \Delta_{\boldsymbol{j}_{x}} & \Delta_{\boldsymbol{j}_{\psi_{y}}} & \Delta_{\boldsymbol{j}_{y}} & \Delta_{\boldsymbol{j}_{\psi_{\boldsymbol{x}}}}
\end{array}\right\}^{\boldsymbol{T}},
$$

where the subscript $j$ denotes each location of interest across the entire host surface. In the case of a single cell there are 4 points of interest, the actuation triangle and the tips of the three prongs $(\mathrm{A}, \mathrm{B}, \mathrm{C})$ within the cell.

Because of the complexity of the host surface due to numerous cell interactions, $\boldsymbol{K}_{\text {net }}$ is not easily obtained directly. However, it can be obtained indirectly using the Matrix Displacement approach as outlined by Pestel and Leckie ${ }^{13}$ by defining $\boldsymbol{K}_{\text {net }}$ in terms of two matrices, the unassembled stiffness matrix, $\boldsymbol{K}_{\boldsymbol{u}}$ and the compatibility matrix, $\boldsymbol{\epsilon}$ :

$$
\boldsymbol{K}_{n e t}=\epsilon^{T} \boldsymbol{K}_{u} \epsilon .
$$

The role of the compatibility matrix is to ensure that the local deformations of all the flexible elements throughout the mechanism, $v$, are consistent with the applied global displacements, $\boldsymbol{D}$, ensuring system integrity under load (Figure5):

$$
v=€ D \text {. }
$$

The transpose of the compatibility matrix relates the externally applied forces, $\boldsymbol{F}$, to the locally defined elemental loads, $\boldsymbol{p}$, using equilibrium constraints:

$$
\boldsymbol{F}=\epsilon^{T} \boldsymbol{p} .
$$

Thus the compatibility matrix, $\boldsymbol{\epsilon}$, contains all the connectivity information for the entire host surface. The unassembled stiffness matrix, $\boldsymbol{K}_{\boldsymbol{u}}$, contains only the locally defined force-deflection relationships for the flexible elements comprising the surface. By isolating the force-deflection information from the connectivity information, the net system stiffness matrix, $\boldsymbol{K}_{\text {net }}$, can be readily computed regardless of surface complexity.

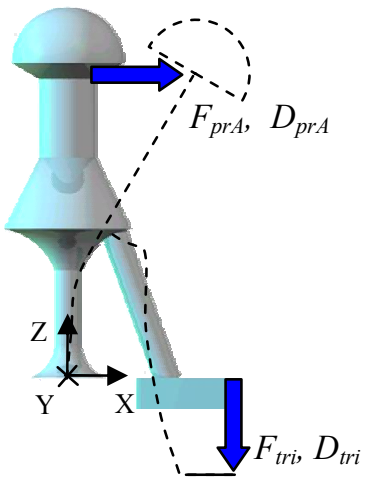

a) Global Forces / Displacements
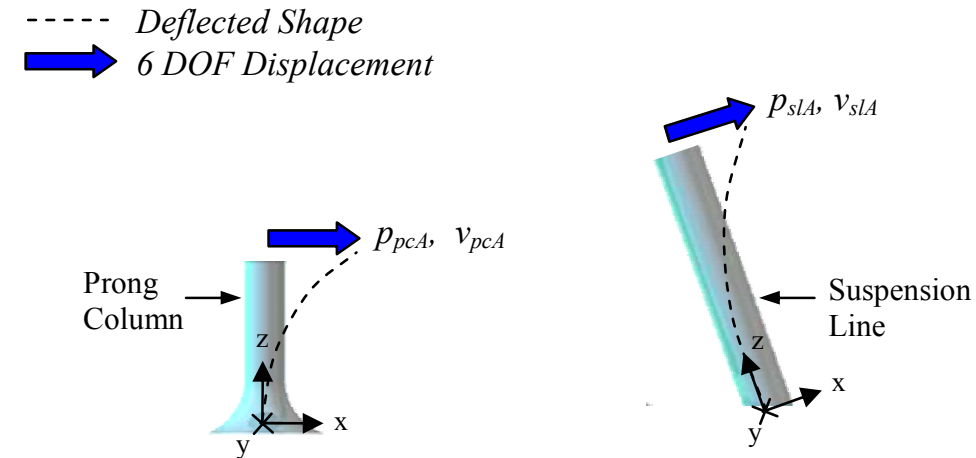

b) Local Loads / Deformations

Figure 5: Global Displacements vs. Local Deformations. (only one prong shown for clarity) a) The model permits the application of globally defined forces and displacements at the actuation triangles ( $\left.\boldsymbol{F}_{\text {tri, }}, \boldsymbol{D}_{\text {tri. }}\right)$ and the center of each prong head $\left(F_{p r A, B, C}, D_{\text {pra,B,C }}\right)$. b) The compatibility equations convert these globally defined forces and displacements into the locally defined loads and deformations at the flexible prong columns and suspension lines $\left(\boldsymbol{p}_{p c A, B, C}, \boldsymbol{v}_{p c A, B, C}, \boldsymbol{p}_{s l A, B, C}, v_{s l A, B, C}\right)$. 


\section{UNASSEMBLED STIFFNESS MATRIX}

The unassembled stiffness matrix, $\boldsymbol{K}_{\boldsymbol{u}}$, is a simple diagonal matrix of the general form:

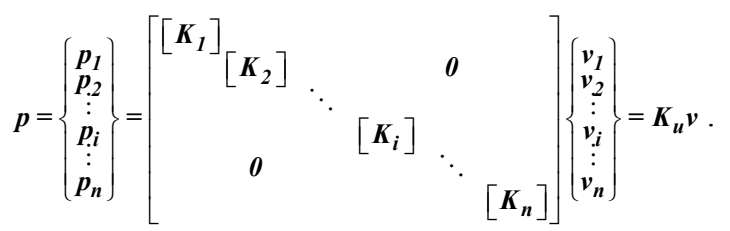

where $\boldsymbol{p}$ is a vector of locally defined load vectors applied to all $n$ flexible elements (prong columns and suspension lines) of the structure and $\boldsymbol{v}$ is a vector of the locally defined displacement vectors of all $n$ flexible elements. Note that $\boldsymbol{K}_{\boldsymbol{u}}$, is a diagonal matrix and thus represents only a collection of unassembled element stiffness matrices $\boldsymbol{K}_{\boldsymbol{i}}$. The unassembled elemental stiffness matrix, $\boldsymbol{K}_{\boldsymbol{i}}$, was derived for each flexible element using classical beam and bar theory for a generalized cantilevered beam assuming that the locally defined loads $\boldsymbol{p}_{\boldsymbol{i}}$ (shears - $V_{x}, V_{y}$, tip moments - $M_{y}, M_{x}$, axial load $-N_{z}$, and axial torque - $T_{z}$ ) act independently and can be decoupled. These loads are related to the locally defined deformations $\boldsymbol{v}_{\boldsymbol{i}}$ (transverse deformations $-w_{x}, w_{y}$, tip rotations $-\psi_{y}, \psi_{x}$, axial deformation $-\mu_{z}$, and twist $-\phi_{z}$, ) through the elemental stiffness matrix $\boldsymbol{K}_{\boldsymbol{i}}$ :

$\boldsymbol{p}_{\boldsymbol{i}}=\left\{\begin{array}{c}N_{z} \\ T_{z} \\ V_{x} \\ M_{y} \\ V_{y} \\ M_{x}\end{array}\right\}=\left[\begin{array}{cccccc}\frac{E \Lambda}{L} & 0 & 0 & 0 & 0 & 0 \\ 0 & \frac{G J}{L} & 0 & 0 & 0 & 0 \\ 0 & 0 & \frac{12 E I}{L^{3}} & \frac{-6 E I}{L^{2}} & 0 & 0 \\ 0 & 0 & \frac{-6 E I}{L^{2}} & \frac{4 E I}{L} & 0 & 0 \\ 0 & 0 & 0 & 0 & \frac{12 E I}{L^{3}} \frac{6 E I}{L^{2}} \\ 0 & 0 & 0 & 0 & \frac{6 E I}{L^{2}} & \frac{4 E I}{L}\end{array}\right]\left\{\begin{array}{c}u_{z} \\ \varphi_{z} \\ w_{x} \\ \psi_{y} \\ w_{y} \\ \psi_{x}\end{array}\right\}=\boldsymbol{K}_{\boldsymbol{i}} \boldsymbol{v}_{\boldsymbol{i}}$

composed of geometry ( $L$ - length of the element, $\Lambda$ cross sectional area, $I$ - area moment of inertia, and $J$ polar moment of inertia) and material parameters $(E$ Young's modulus, $G$ - shear modulus). Since the boundary conditions and material for the prong columns and suspension lines are identical, their elemental stiffness matrices differ only in the geometric parameters.

\section{COMPATIBILITY MATRIX}

The compatibility matrix, $\boldsymbol{\epsilon}$, holds all the connection information and thus is dependent on the specific formation of elements comprising the structure. It will be different depending on how many cells are considered. The form of the single-cell compatibility matrix is presented here; however, the symmetry in the structure allows this solution to be expanded easily to capture the behavior of any number of cells, as shown in the next section.

For each cell under general loading conditions, $\boldsymbol{F}$, a 36 x 24 compatibility matrix, $\boldsymbol{\epsilon}$, is necessary to fully define the relationship between the global displacements, $\boldsymbol{D}$, at the tip of each prong and actuation triangle and the local element deformations, $\boldsymbol{v}$, within each prong column and suspension line:

$$
\begin{aligned}
& v=€ D, \\
& v=\left\{\begin{array}{llllll}
v_{s l A} & v_{p c A} & v_{s l B} & v_{p c B} & v_{s l C} & v_{p c C}
\end{array}\right\}^{T}, \\
& D=\left\{\begin{array}{llll}
D_{t r i} & D_{p r A} & D_{p r B} & D_{p r C}
\end{array}\right\}^{T}, \\
& \epsilon=\left[\begin{array}{cccc}
{\left[\epsilon_{s l A, t r i}\right]} & {\left[\epsilon_{s l A, p r A}\right]} & 0 & 0 \\
0 & {\left[\epsilon_{p c A, p r A}\right]} & 0 & 0 \\
{\left[\epsilon_{s l B, t r i}\right]} & 0 & {\left[\epsilon_{s l B, p r B}\right]} & 0 \\
0 & 0 & {\left[\epsilon_{p c B, p r B}\right]} & 0 \\
{\left[\epsilon_{s l C, t r i}\right]} & 0 & 0 & {\left[\epsilon_{s l C, p r C}\right]} \\
0 & 0 & 0 & {\left[\epsilon_{p c C, p r C}\right]}
\end{array}\right]
\end{aligned}
$$

where $\boldsymbol{\epsilon}_{i, j}$ refers to the 6 × 6 compatibility sub-matrix for "ith flexible element" with respect to globally defined displacements of the " $\mathrm{j}$ th location".

These sub-matrices are derived by enforcing a virtual displacement of a single degree of freedom within the displacement vector, $\boldsymbol{D}$, while holding all other displacements fixed at zero and examining the resulting locally defined deformation of the flexible elements, $\boldsymbol{v}$. This process is greatly simplified by the purely local effects of the virtual displacement theorem which allows for the rapid recognition of empty compatibility sub-matrices since it is generally obvious which flexible elements are rigidly tied to each global degree of freedom. For example, if the chosen virtual displacement is within the $\boldsymbol{D}_{\boldsymbol{p} \boldsymbol{r} \boldsymbol{A}}$ vector, no deformations of prong columns $\mathrm{B}$ and $\mathrm{C}$ or suspension lines $\mathrm{B}$ and $\mathrm{C}$ are generated resulting in the 4 empty sub-matrices in the second column of $\boldsymbol{\epsilon}$.

The nine non-zero sub-matrices $\left(\boldsymbol{\epsilon}_{p c A, B, C, p r A, B, C}, \boldsymbol{\epsilon}_{s l A, B, C}\right.$, $\left.p r A, B, C, \boldsymbol{\epsilon}_{s l A, B, C, t r i}\right)$ can be reduced down to three unique sub-matrices $\left(\boldsymbol{\epsilon}_{p c, p r}, \boldsymbol{\epsilon}_{s l, p r}, \boldsymbol{\epsilon}_{s l, t r i}\right)$ using the symmetry of the prong cell. Only simple global Z-axis rotations about the center of the actuation triangle, $\boldsymbol{R}_{\boldsymbol{B}}$ and $\boldsymbol{R}_{\boldsymbol{C}}$, applied through angles $\beta_{B}=-120^{\circ}$ and $\beta_{C}=120^{\circ}$, are required to define the sub-matrices for prong columns/suspension lines $\mathrm{B}$ and $\mathrm{C}$ in terms of the submatrices for prong columns/suspension lines A: 


$$
\epsilon=\left[\begin{array}{cccc}
{\left[\epsilon_{s L A, t r i}\right]} & {\left[\epsilon_{s L A, p r A}\right]} & 0 & 0 \\
0 & {\left[\epsilon_{p c A, p r A}\right]} & 0 & 0 \\
{\left[\epsilon_{S L A, t r i} R_{B}\right]} & 0 & {\left[\epsilon_{S l A, p r A} R_{B}\right]} & 0 \\
0 & 0 & {\left[\epsilon_{p c A, p r A} R_{B}\right]} & 0 \\
{\left[\epsilon_{s L A, t r i} R_{C}\right]} & 0 & 0 & {\left[\epsilon_{s L, p r A} R_{C}\right]} \\
0 & 0 & 0 & {\left[\epsilon_{p c A, p r A} R_{C}\right]}
\end{array}\right]
$$

The sub-matrix $\boldsymbol{\epsilon}_{\boldsymbol{p} \boldsymbol{A} \boldsymbol{p} \boldsymbol{p r} \boldsymbol{A}}$ converts the globally defined displacements at the tip of prong A, $\boldsymbol{D}_{\text {prA }}$, into the locally defined deformations of flexible prong column $\mathrm{A}, v_{p c A}$, based on the geometry of the undeformed cell:

$$
\begin{gathered}
\boldsymbol{v}_{p c A}=\boldsymbol{\epsilon}_{p c A, p r A} \boldsymbol{D}_{p r A}, \\
\boldsymbol{v}_{p c A}=\left\{u_{p c A_{z}} \phi_{p c A_{z}} w_{p c A_{x}} \psi_{p c A_{y}} w_{p c A_{y}} \psi_{p c A_{x}}\right\}^{T}, \\
\boldsymbol{D}_{p r A}=\left\{\Delta_{p r A_{z}} \Delta_{p r A_{q_{z}}} \Delta_{p r A_{x}} \Delta_{p r A_{\psi_{y}}} \Delta_{p r A_{y}} \Delta_{p r A_{\psi_{x}}}\right\}^{T}, \\
\boldsymbol{\epsilon}_{p c A, p r A}=\left[\begin{array}{cccccc}
1 & 0 & 0 & 0 & 0 & 0 \\
0 & 1 & 0 & 0 & 0 & 0 \\
0 & 0 & 1 & -L_{f}-L_{b} & 0 & 0 \\
0 & 0 & 0 & 1 & 0 & 0 \\
0 & 0 & 0 & 0 & 1 & L_{f}+L_{b} \\
0 & 0 & 0 & 0 & 0 & 1
\end{array}\right] .
\end{gathered}
$$

Suspension line A is directly connected to both the actuation triangle and prong $\mathrm{A}$, thus two sub-matrices are required. $\boldsymbol{\epsilon}_{s L A, p r A}$ converts the globally defined displacements of prong A, $\boldsymbol{D}_{p r A}$, into the locally defined deformations of suspension line A, $\boldsymbol{v}_{\boldsymbol{s} I A}$. Similarly, $\boldsymbol{\epsilon}_{\boldsymbol{s} L \boldsymbol{A}}$, tri converts the globally defined displacements of the actuation triangle, $\boldsymbol{D}_{t r i}$, into the locally defined deformations of suspension line A, $\boldsymbol{v}_{\boldsymbol{s l A}}$ :

$$
\begin{aligned}
& v_{S L A}=\epsilon_{S L A, p r A} D_{p r A}, \\
& v_{s l A}=\epsilon_{s l A, t r i} D_{t r i}, \\
& v_{s l A}=\left\{\begin{array}{llllll}
u_{s l A_{z}} & \phi_{s l A_{z}} & w_{s l A_{x}} & \psi_{s l A_{y}} & w_{s l A_{y}} & \psi_{s l A_{x}}
\end{array}\right\}^{T}, \\
& \boldsymbol{D}_{t r i}=\left\{\Delta_{t r i_{\mathrm{z}}} \Delta_{t r i_{\varphi_{z}}} \Delta_{t r r_{\mathrm{x}}} \Delta_{t r i_{\psi_{\mathrm{y}}}} \Delta_{t r i_{\mathrm{y}}} \Delta_{t r r_{\psi_{x}}}\right\}^{T},
\end{aligned}
$$

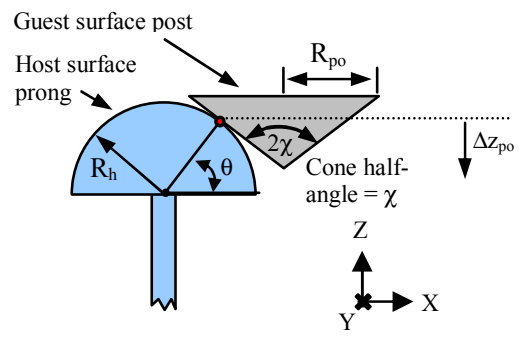

a) Initial Contact

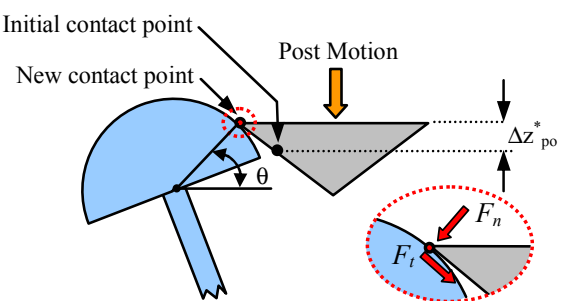

b) Transition Point

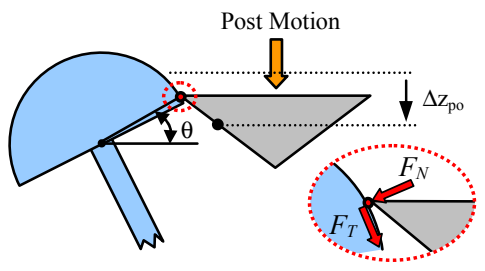

c) Post-Transition

Figure 6: Engagement Phases. (only one prong shown for clarity) a) The location of the initial contact point is a function of the post shape, prong shape, and the diameter of the prong head. At this point the frictional loads are tangent to both the post and prong lead surfaces and remain tangent until the transition point is reached. b) A transition in the engagement behavior occurs when the maximum radius of the guest surface post, $R_{p o}$, becomes the contact point between the post and prong. c) After the incoming post passes the transition point, the posts and prongs are no longer tangent to each other and thus frictional loads are no longer tangent to the post. Engagement behavior is no longer defined by the shape of the postís lead surface but rather the shape of the prongís lead surface. 
longer mutually tangent. Since the contact point on the post remains fixed at the maximum diameter after the transition, the engagement behavior switches from being defined by the shape of the post's lead surface to the shape of the prong's lead surface.

For this model, the engaging post is assumed to enter the cell vertically and centered, distributing any loads evenly amongst the three prongs of the cell. Since the cell is axi-symmetric about the actuation triangle, the number of degrees of freedom of the mechanism can be reduced to 4 by only considering the actuation triangle and a single prong:

$$
\begin{gathered}
\boldsymbol{F}^{\text {sym }}=\left(\boldsymbol{\epsilon}^{\text {sym }}\right)^{\boldsymbol{T}} \boldsymbol{K}_{\boldsymbol{u}}^{\boldsymbol{s y m}} \boldsymbol{\epsilon}^{s y \boldsymbol{m}} \boldsymbol{D}^{\text {sym }}, \\
\boldsymbol{F}^{\text {sym }}=\left\{\begin{array}{llll}
F_{p r A_{z}} & F_{p r A_{x}} & M_{p r A_{y}} & F_{t r r_{z}}
\end{array}\right\}^{\boldsymbol{T}}, \\
\boldsymbol{D}^{\text {sym }}=\left\{\begin{array}{llll}
\Delta_{p r A_{z}} & \Delta_{p r A_{x}} & \Psi_{p r A_{y}} & \Delta_{t r i_{z}}
\end{array}\right\}^{\boldsymbol{T}} .
\end{gathered}
$$

Only loads and deformations in the XZ plane are required thus simplifying the unassembled matrix (Equation 6) and compatibility matrix (Equation 9) considerably to $\boldsymbol{K}_{u}^{\text {sym }}$ and $\boldsymbol{\epsilon}^{\text {sym }}$ :

$$
\begin{aligned}
& p_{u}^{s y m}=K_{u}^{s y m} v_{u}^{s y m} \\
& \boldsymbol{p}_{\boldsymbol{u}}^{\text {sym }}=\left\{\begin{array}{llllll}
N_{s l} & V_{s l_{x}} & M_{s l_{y}} & N_{p c} & V_{p c_{x}} & M_{p c_{y}}
\end{array}\right\}^{T}, \\
& \boldsymbol{v}_{\boldsymbol{u}}^{\text {sym }}=\left\{\begin{array}{llllll}
u_{z s l} & w_{x s l} & \psi_{y s l} & u_{z p c} & w_{x p c} & \psi_{y p c}
\end{array}\right\}^{T}, \\
& \boldsymbol{K}_{\boldsymbol{u}}^{\boldsymbol{s y m}}=\left[\begin{array}{cccccc}
\frac{E \Lambda_{s l}}{L_{s l}} & 0 & 0 & 0 & 0 & 0 \\
0 & \frac{12 E I_{s l}}{L_{s l}^{3}} \frac{-6 E I_{s l}}{L_{s l}^{2}} & 0 & 0 & 0 \\
0 & \frac{-6 E I_{s l}}{L_{s l}^{2}} \frac{4 E I_{s l}}{L_{s l}} & 0 & 0 & 0 \\
0 & 0 & 0 & \frac{E \Lambda_{p c}}{L_{p c}} & 0 & 0 \\
0 & 0 & 0 & 0 & \frac{12 E I_{s l}}{L_{p c}^{3}} & \frac{-6 E I_{p c}}{L_{p c}^{2}} \\
0 & 0 & 0 & 0 & \frac{-6 E I_{p c}}{L_{p c}^{2}} & \frac{4 E I_{p c}}{L_{p c}}
\end{array}\right] \\
& {\left[\begin{array}{cccccc}
a_{s l} & 0 & 0 & 0 & 0 & 0 \\
0 & c_{s l} & d_{s l} & 0 & 0 & 0
\end{array}\right]} \\
& \begin{array}{llllll}
0 & d_{s l} & e_{s l} & 0 & 0 & 0
\end{array} \\
& =\begin{array}{llllll}
0 & 0 & 0 & a_{p c} & 0 & 0
\end{array} \\
& \begin{array}{llllll}
0 & 0 & 0 & 0 & c_{p c} & d_{p c} \\
0 & 0 & 0 & 0 & d_{p c} & \\
0 & 0 &
\end{array} \\
& {\left[\begin{array}{llllll}
0 & 0 & 0 & 0 & d_{p c} & e_{p c}
\end{array}\right]} \\
& \boldsymbol{\epsilon}^{\text {sym }}=\left[\begin{array}{cccc}
1 & 0 & 0 & 0 \\
0 & 1 & -L_{\mathrm{f}}-L_{b} & 0 \\
0 & 0 & 1 & 0 \\
\sin (\alpha) & -\cos (\alpha) & L_{b} \cos (\alpha)-R_{b} \sin (\alpha) & -\sin (\alpha) \\
\cos (\alpha) & \sin (\alpha) & -R_{b} \cos (\alpha)-L_{b} \sin (\alpha) & -\cos (\alpha) \\
0 & 0 & 1 & 0
\end{array}\right] .
\end{aligned}
$$

During engagement it is assumed that the SMA is not activated and the actuation triangle vertical force, $F_{t r i z}$, is zero. The other external loads, $F_{p r A_{x}}, F_{p r A_{z}}$, and moment, $M_{\text {prAy }}$, are applied to the prong as a result of both the normal, $F_{N}$, and tangential, $F_{T}$, loads applied by the engaging post. To achieve a closed form solution, coulomb friction was assumed $\left(F_{T}=\mu_{E} F_{N}\right)$ resulting in the following globally defined loads applied to the prong,

$$
\begin{aligned}
& F_{p r A_{z}}=F_{N}\left[-\sin (\theta)-\mu_{E} \cos (\theta)\right] \\
& F_{p r A_{x}}=F_{p r A_{z}} \frac{\left[-\cos (\theta)+\mu_{E} \sin (\theta)\right]}{\left[-\sin (\theta)-\mu_{E} \cos (\theta)\right]}, \\
& M_{p r A_{y}}=F_{p r A_{z}} \frac{\mu_{E} R_{h}}{\left[-\sin (\theta)-\mu_{E} \cos (\theta)\right]}
\end{aligned}
$$

where $R_{h}$ is the prong head radius, $\theta$ is the contact angle measured from the horizontal plane to the contact point, and $\mu_{E}$ is the coefficient of friction between the post and prong lead surfaces. Thus the load vector, $\boldsymbol{F}$, can be reduced to a single unknown, $F_{p r A_{z}}$, leaving five unknowns $\left(F_{p r A_{z}}, \Delta_{p r A_{z}}, \Delta_{p r A_{x}}, \Delta_{p r A \psi_{y}}, \Delta_{t r i_{z}}\right)$ with four equations captured by Equation 13 .

The horizontal prong displacement, $\Delta_{p r A_{x}}$, can be related to the vertical distance traveled by the engaging post relative to the initial contact point, $\Delta z_{p o}$. Since the conical post and hemispherical prong remain mutually tangent up to the transition point of Figure $6 \mathrm{~b}$, the contact angle, $\theta$, is defined solely by the post's conical half angle. After the transition point has been passed, the contact angle is a function of the current post position, $\Delta z_{p o}$, relative to its position at the transition point, $\Delta z^{*}{ }_{p o}$ :

$$
\begin{aligned}
& \theta=\left\{\begin{array}{cc}
\chi & 0>\Delta z_{p o}>\Delta z_{p o}^{*} \\
\sin ^{-1}\left(\frac{\Delta z_{p o}-\Delta z_{p o}^{*}+R_{h} \sin (\chi)}{R_{p r}}\right), & \Delta z_{p o}<\Delta z_{p o}^{*}
\end{array}\right\} \\
& \Delta z_{p o}^{*}=\frac{-R_{p o}+R_{\text {cell }}-R_{h} \cos (\chi)}{\tan (\chi)} .
\end{aligned}
$$

Because the post has an idealized conical shape, the horizontal prong displacement for prong $\mathrm{A}, \Delta_{p r A_{x}}$, is a simple function of the vertical post displacement, $\Delta z_{p o}$. Just as with the expression for the contact angle, the expression defining how the prongs are deflected in response to an incoming post is also distinct over two ranges: from initial contact up to the transition point and from the transition point until engagement is completed:

$$
\Delta_{p r A_{x}}=\left\{\begin{array}{cc}
\Delta z_{p o} \tan (\chi) \quad, & 0>\Delta z_{p o}>\Delta z_{p o}^{*} \\
-R_{h} \cos (\theta)+\left[R_{\text {cell }}-R_{p o}\right], & \Delta z_{p o}<\Delta z_{p o}^{*}
\end{array}\right\} .
$$




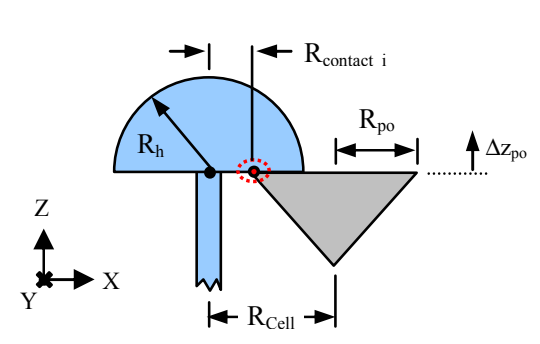

a) Initial Engaged Post Position

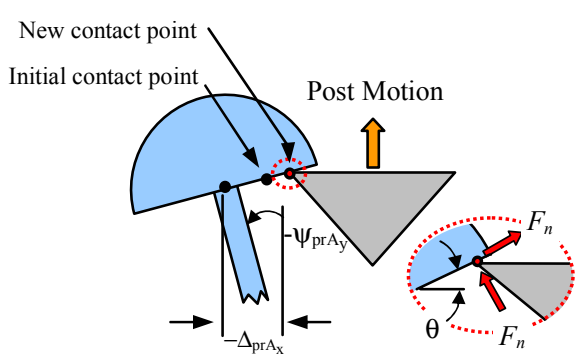

b) Midway Through Separation

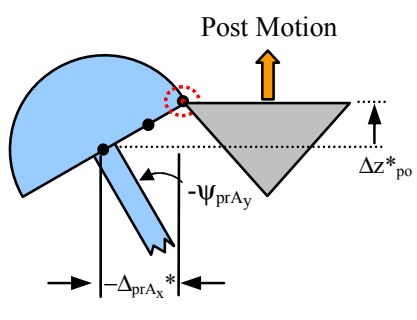

c) Separation Point

Figure 7: Retention. (only one prong shown for clarity) a) Initially the trailing surfaces of the post and prongs are parallel. The initial contact point, $R_{\text {contact_ } i}$ is determined by the radius of the post, $R_{\text {po, }}$ and the radius of the cell, $R_{\text {cell. }}$ b) As the post retreats, the contact point slides towards the edge of the prong connection topology. c) Separation occurs when the post clears the prong.

By substituting in Equation 19 for $\Delta_{p r A_{x}}$, the global equation 13 can be solved for the engagement force, $F_{E}$, as a function of the post displacement, $\Delta z_{p o}$ :

$$
F_{E}=3 F_{p r A_{z}}=\left\{\begin{array}{cc}
\Delta z_{p o} \tan (\chi) \frac{N_{E}}{D_{E}} \quad, \quad 0>\Delta z_{p o}>\Delta z_{p o}^{*} \\
\left(R_{\text {cell }}-R_{p o}-R_{h} \cos (\theta)\right) \frac{N_{E}}{D_{E}}, & \Delta z_{p o}<\Delta z_{p o}^{*}
\end{array}\right\},
$$

where,

$$
\begin{aligned}
& N_{E}=3\left[\begin{array}{l}
-c_{s l} d_{p c}^{2}+c_{p c}\left\{-d_{s l}^{2}+c_{s l}\left(e_{s l}+e_{p c}\right)\right\}+ \\
a_{s l}\left\{-2 d_{s l}^{2}-d_{p c}^{2}+\left(2 c_{s l}+c_{p c}\right)\left(e_{s l}+e_{p c}\right)-4 c_{s l} d_{p c} L_{f}+2 c_{s l} c_{p c} L_{f}^{2}\right\} \\
-\cos (2 \alpha)\left[\left(-a_{s l}+c_{s l}\right) d_{p c}^{2}+c_{p c}\left\{d_{s l}^{2}+\left(a_{s l}-c_{s l}\right)\left(e_{s l}+e_{p c}\right)\right\}\right] \\
-4 a_{s l} d_{s l}\left(d_{p c}-c_{p c} L_{f}\right) \sin (\alpha)
\end{array}\right] \\
& D_{E}=\frac{F_{x}}{F_{z}} *\left[\begin{array}{l}
d_{s l}^{2}-\left(a_{s l}+c_{s l}\right)\left(e_{s l}+e_{p c}-2 d_{p c} L_{f}+c_{p c} L_{f}^{2}\right)+ \\
L_{b}\left\{2\left(a_{s l}+c_{s l}\right)\left(d_{p c}-c_{p c} L_{f}\right)\right\} \\
-L_{b}^{2}\left\{2 a_{s l} c_{s l}+\left(a_{s l}+c_{s l}\right) c_{p c}\right\}
\end{array}\right]+ \\
& \frac{M_{y}}{F_{z}}\left[\left(a_{s l}+c_{s l}\right)\left(d_{p c}-c_{p c} L_{f}\right)-L_{b}\left\{2 a_{s l} c_{s l}+\left(a_{s l}+c_{s l}\right) c_{p c}\right\}\right]+ \\
& 2 a_{s l} d_{s l}\left(2 \frac{F_{x}}{F_{z}} L_{b}+\frac{M_{y}}{F_{z}}\right) \sin (\alpha)+\cos (2 \alpha) \square \\
& {\left[\begin{array}{l}
d_{s l}^{2} \frac{F_{x}}{F_{z}}+\left(a_{s l}-c_{s l}\right) \\
\left\{\begin{array}{l}
\frac{F_{x}}{F_{z}}\left\{e_{s l}+e_{p c}+\left(L_{f}+L_{b}\right)\left\{-2 d_{p c}+c_{p c}\left(L_{f}+L_{b}\right)\right\}\right\}+ \\
\frac{M_{y}}{F_{z}}\left\{-d_{p c}+c_{p c}\left(L_{f}+L_{b}\right)\right\}
\end{array}\right\}
\end{array}\right]}
\end{aligned}
$$

Although Equation 20 appears rather complicated at first glance, the majority of the terms are simply dimensions of the mechanism. Only the load ratios, $F_{p r A x} / F_{p r A_{z}}$ and $M_{p r A y} / F_{p r A_{z}}$ vary throughout the engagement process as the contact angle changes according to Equation 17 thereby altering the directions of the normal and frictional applied loads and in turn the engagement force.

\section{RETENTION MODEL}

Once the guest surface engages the host surface it is retained by the trailing surfaces of the post and prong. The initial contact location, $R_{\text {contact } i}$, on the prong is defined as the difference between the radius of the cell, $R_{\text {cell }}$, and the maximum radius of the post connection topology, $R_{p o}$. As the post begins to separate from the prong cell, the tip of the post $\left(R_{p o}\right)$ translates along the trailing surface of the prong, causing the prong to deflect outward with a horizontal displacement, $\Delta_{p r A_{x}}$, as in Figure 7. Release of the post from the prong cell is achieved when the post tip reaches the outer radius of the prong, $R_{h}$ or

$$
-\Delta_{p r A_{x}}^{*}=R_{h}-R_{\text {contact_i }_{-}}
$$

The net stiffness matrix and compatibility matrix for retention are the same as for engagement (Equations 14 and 15) but naturally the loads applied to the prongs are different. The loads are transferred from the retreating post equally to the prongs through both the normal force, $F_{N}$, and the coulomb friction force $F_{T}=\mu_{R} F_{N}$ :

$$
\begin{aligned}
& F_{p r A_{z}}=F_{N}\left[\cos (\theta)+\mu_{R} \sin (\theta)\right] \\
& F_{p r A_{x}}=F_{p r A_{z}} \frac{\left[-\sin (\theta)+\mu_{R} \cos (\theta)\right]}{\left[\cos (\theta)+\mu_{R} \sin (\theta)\right]} . \\
& M_{\text {prA }_{y}}=F_{p r A_{z}} \frac{-R_{\text {contact_i }} i}{\left[\cos (\theta)+\mu_{R} \sin (\theta)\right]}
\end{aligned}
$$

The contact angle, $\theta$, is measured from the horizontal plane to the contact point and equal to the negative of the deflected prong angle, $\psi_{p r A y}$, which varies through the retention cycle. Similar to engagement, this reduces the global system of equations (Equation 13) leaving five unknowns $\left(F_{p r A_{z}}, \Delta_{p r A_{z}}, \Delta_{p r A_{x}}, \Delta_{p r A \psi_{y}}, \Delta_{t r i_{z}}\right)$. This can be solved to cast the retention force as a function of $\psi_{\text {prAy }}$ :

$$
F_{R}=\psi_{\text {prA }_{y}} \frac{N_{R}}{D_{R}}
$$


where,

$$
N_{R}=3\left[\begin{array}{l}
c_{s l} d_{p c}^{2}+c_{p c}\left\{d_{s l}^{2}-c_{s l}\left(e_{s l}+e_{p c}\right)\right\}+ \\
a_{s l}\left\{2 d_{s l}^{2}+d_{p c}^{2}-\left(2 c_{s l}+c_{p c}\right)\left(e_{s l}+e_{p c}\right)+4 c_{s l} d_{p c} L_{f}-2 c_{s l} c_{p c} L_{f}^{2}\right\}+ \\
\cos (2 \alpha)\left[\left(-a_{s l}+c_{s l}\right) d_{p c}^{2}+c_{p c}\left\{d_{s l}^{2}+\left(a_{s l}-c_{s l}\right)\left(e_{s l}+e_{p c}\right)\right]\right]+ \\
4 a_{s l} d_{s l}\left(d_{p c}-c_{p c} L_{f}\right) \sin (\alpha)
\end{array}\right]
$$$$
\mathrm{D}_{\mathrm{R}}=\frac{F_{x}}{F_{z}}\left[\left(a_{s l}+c_{s l}\right)\left(d_{p c}-c_{p c} L_{f}\right)-L_{b}\left\{2 a_{s l} c_{s l}+c_{p c}\left(a_{s l}+c_{s l}\right)\right\}\right]-
$$$$
\frac{M_{y}}{F_{z}}\left[2 a_{s l} c_{s l}+\left(a_{s l}+c_{s l}\right) c_{p c}\right]-
$$$$
\left.\cos (\alpha)\left[\left(a_{s l}-c_{s l}\right)\left\{d_{p c} \frac{F_{x}}{F_{z}}-c_{p c} \frac{F_{x}}{F_{z}}\left(L_{f}+L_{b}\right)+\frac{M_{y}}{F_{z}}\right)\right\}\right]+
$$$$
\frac{\mathrm{F}_{\mathrm{x}}}{\mathrm{F}_{\mathrm{z}}}\left[2 \mathrm{a}_{\mathrm{sl}} \mathrm{d}_{\mathrm{sl}} \sin (\alpha)\right]
$$

$\psi_{\text {prAy }}$ is bounded by:

$$
0 \leq \psi_{p r A_{y}} \leq \psi_{p r A_{y}}^{*},
$$

where $\psi^{*}{ }_{p r A y}$ is the angular rotation of prong $\mathrm{A}$ at the point of guest surface separation occurring at $\Delta_{p r A_{x}}^{*}$ given in Equation 21. Typically the angular displacement, $\psi_{\text {pray }}$, is not of significant interest; rather it is more desirable to relate the retention force, $F_{R}$, to the displacement of the post, $\Delta z_{p o}$. Applying the small angle approximation for the angular prong rotation $\psi_{p r A_{y}}$, the vertical post displacement $\Delta z_{p o}$ can defined in terms of the initial contact radius, $R_{\text {contact_ }}$, the horizontal prong displacement, $A_{p r A_{x}}$, and $\psi_{p r A_{y}}$ :

$$
\Delta z_{p o}=-\left(R_{\text {contact }_{-} i}-\Delta_{p r A_{x}}\right) \psi_{p r A_{y}} .
$$

Following the solution of Equation 13, $\Delta_{p r A_{x}}$, is known and Equation 25 can be solved. Separation occurs in one smooth motion, unlike engagement where there were two distinct stages. The only guest surface parameter impacting the retention behavior is the outer radius of the post, $R_{p o}$, which has a two-fold impact. First it determines how far the prongs must be deflected before separation occurs (Equation 21). Second it impacts the moments imparted on the prongs, $M_{\text {prAy }}$, as the guest surface tries to separate from the host surface (Equation 22). Thus as the $R_{p o}$ increases the moments trying to deflect the prongs are reduced, increasing retention force, and the vertical post distance at transition is increased, further raising the retention force.

\section{MODEL EXPANSION}

Because the host surface is comprised of a repeated pattern of identical cells, it is straightforward to expand the single-cell model to a multi-cell model. Adding cells does not linearly increase the degrees of freedom for the surface because prongs are shared between adjacent cells. For example, a three-cell host surface (Figure 2) adds three displacement vectors for the new actuation triangle and two new prongs $\left(\boldsymbol{D}_{t r i 2}, \boldsymbol{D}_{p r A_{2}}\right.$ and $\boldsymbol{D}_{p r B_{2}}$ ) instead of four because prong B1 and C2 are the same.

The compatibility matrix, $\boldsymbol{\epsilon}$, converts these new displacements and those of the original single cell into the deformations of all the flexible elements in the surface, $v$ (Equation 26 below).

The shaded portion of Equation 26 is exactly the compatibility matrix for the single-cell case (Equation 9), while the remainder of $\boldsymbol{\epsilon}$ contains sub-matrices for the new flexible prong columns and suspension lines. Application of the virtual displacement theory quickly identifies empty $\boldsymbol{\epsilon}_{i j}$ sub-matrices as it did in the singlecell model development. For example, displacing prong $A_{1}$ alone has no impact on the added prong columns and suspension lines thus all of the added submatrices in the second column of $\boldsymbol{\epsilon}$ are empty. However, displacing prong $\mathrm{B}_{1}$, will directly cause the

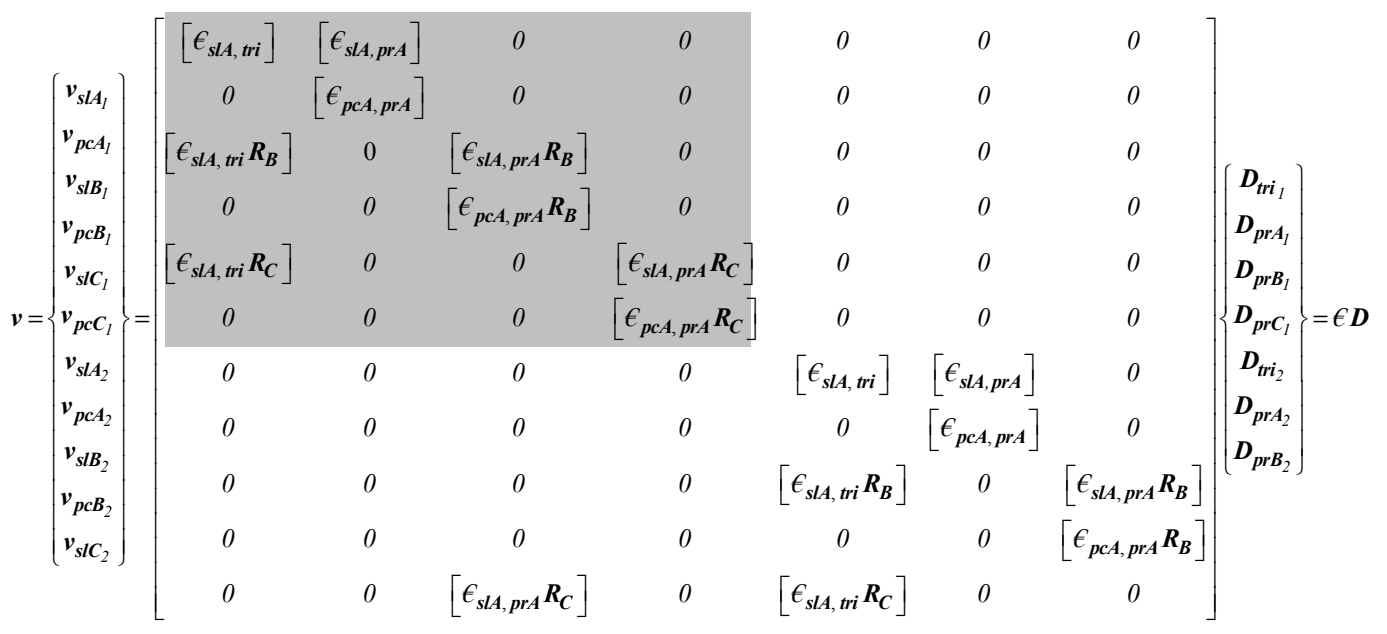




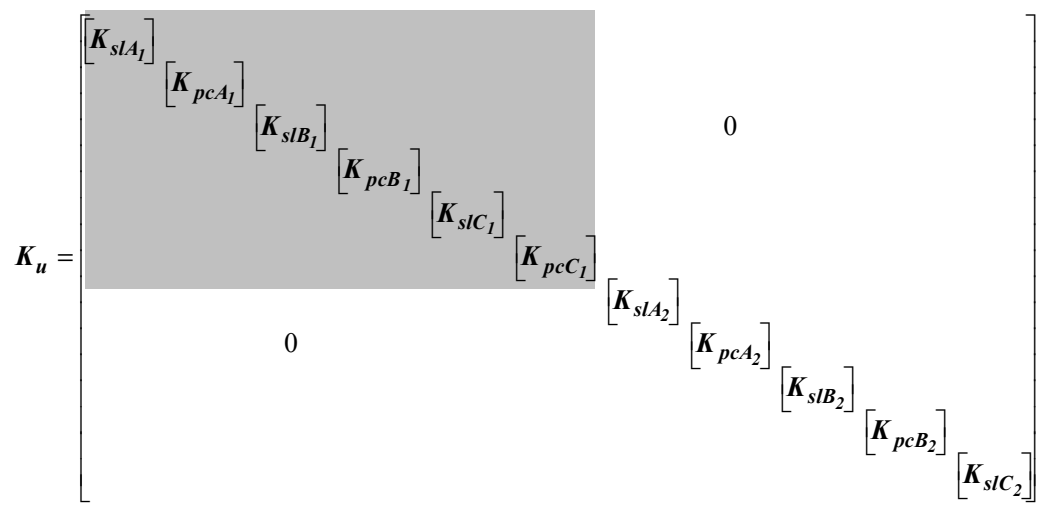

deformation of suspension line $\mathrm{C}_{2}\left(\boldsymbol{v}_{\boldsymbol{s}_{2} \boldsymbol{C}_{2}}\right)$ since it is shared; thereby necessitating the placement of the submatrix $\boldsymbol{\epsilon}_{\text {slA, prA }} \boldsymbol{R}_{\boldsymbol{C}}$ into the third column of $\boldsymbol{\epsilon}$. Since all cells are identical, no new sub-matrices, $\boldsymbol{\epsilon}_{i j}$, need to be derived to complete $\boldsymbol{\epsilon}$, rather Equations 9-12 can be used.

In a similar manner, the unassembled stiffness matrix, $\boldsymbol{K}_{\boldsymbol{u}}$, (Equation 27 above) is expanded by including an additional $\boldsymbol{K}_{\boldsymbol{i}}$ sub-matrix (Equation 7 ) for each of the new flexible elements.

As with the compatibility matrix, this does not grow linearly since $\boldsymbol{K}_{\boldsymbol{p} \boldsymbol{c}_{2}}=\boldsymbol{K}_{\boldsymbol{p} \boldsymbol{c} \boldsymbol{B}_{1}}$. Likewise, every prong column is identical and every suspension line is identical; therefore $\boldsymbol{K}_{\boldsymbol{u}}$ only has two unique submatrices, one for the prong column and one for the suspension line (Equation 7).

Combining the compatibility and unassembled stiffness matrices, yields the force-deflection relationship, in this case for a three-cell host surface:

$$
F=K_{n e t} D,
$$

where $F=\left\{\begin{array}{lllllll}F_{t r i_{1}} & F_{p r A_{1}} & F_{p r B_{1}} & F_{p r C_{1}} & F_{t r i_{2}} & F_{p r A_{2}} & F_{p r B_{2}}\end{array}\right\}^{T}$,

$$
\begin{aligned}
& D=\left\{\begin{array}{llllllll}
D_{t r i_{1}} & D_{p r A_{1}} & D_{p r B_{1}} & D_{p r C_{1}} & D_{t r i_{2}} & D_{p r A_{2}} & D_{p r B_{2}}
\end{array}\right\}^{T} \text {, } \\
& \boldsymbol{K}_{\text {net }}=\epsilon^{T} \boldsymbol{K}_{\boldsymbol{u}} \epsilon .
\end{aligned}
$$

A three-cell surface is the basic configuration necessary for motion generation. While the modeling of motion generation is beyond the scope of this paper, the presented three-cell model does provide insight into the maximum positioning force that the prongs can impose on the post to move it to the next cell. This positioning force is defined as the horizontal force at the tip of the shared prong $\mathrm{B}_{1}, F_{p r B_{1 x}}$. Because the guest surface post remains in contact with prong $\mathrm{B}_{1}$ during positioning, its displacement is equal to the $\mathrm{X}$-axis displacement of prong $\mathrm{B}_{1}, \Delta_{p r B}$. All externally applied loads with the exception of $F_{p r B l x}$ and the vertical forces on the actuation triangles; $F_{\text {tri } I z}$ and $F_{\text {trizz }}$ are set to zero. To simulate the activation of SMA wires, the actuation triangles are displaced a known distance $\Delta_{t r i}$ and $\Delta_{t r i 2 z}$. By varying $\Delta_{p r B}{ }_{1 x}$, the positioning force as a function of guest surface displacement is defined by solution of Equation 28. It is important to note that this model is only an upper bound on the force and displacement. It does not take into account the normal and friction forces between the host prongs and the guest surface post, which will reduce the positioning force. Therefore, this is representative of the positioning of a guest with a small post support column.

\section{EXPERIMENTAL MODEL VALIDATION}

A series of experimental tests were conducted to progressively explore the accuracy of the analytical model using three operational scenarios: single-cell free deflection, three-cell positioning, and single-cell engagement/retention. To begin, the free deflection of a single-cell surface was validated without considering the interactions between cells or the effects of friction. The cell interactions were examined in the positioning force experiments with a three-cell host surface and the frictional effects were examined during the engagement and retention of a guest surface into a single-cell host surface.

\section{Test SAMPLES}

For these experiments two topological designs (Figure 8) were used for both single-cell and three-cell host surface test samples. Design 1 was based on the reduced-scale test bed examined in previous research ${ }^{12}$ with $18 \mathrm{~mm}$ tall prongs spaced $6.35 \mathrm{~mm}$ from the center of the actuation triangle. Design 2 was the result of optimization using the analytical model to reduce stress levels while maintaining the same prong spacing and reducing the prong height. Design 2 uses longer suspension lines and prong columns while reducing the overall prong height to only $13 \mathrm{~mm}$. Other changes in geometry are presented in Figure 8. Examination of the single cell engagement and retention behaviors were accomplished using two guest surface designs each with the same radius, $R_{p o}=4.7 \mathrm{~mm}$, but differing lead surface half-angles, $60^{\circ}$ and $45^{\circ}$ (Figure 9). 


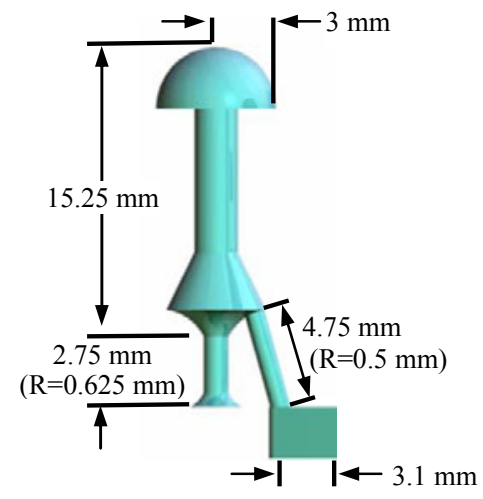

a) Design 1

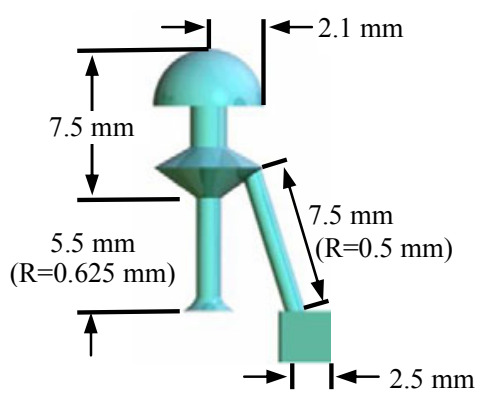

b) Design 2

Figure 9: Experimental Test Sample Designs. Two different designs were fabricated for experimental testing. a) Design 1 is modeled after the prototype tested in prior research ${ }^{12}$ b) Design 2 is the result of refinement to reduce the prongsí height while maintaining the same prong spacing and reducing stress levels.

All guest and host surfaces were fabricated with a 3DSystems SLA-250/40 stereolithography machine using Somos 8110 resin (Young's modulus $\sim 317 \mathrm{MPa}$, flexural modulus $\sim 310 \mathrm{MPa}$, yield stress $\sim 18 \mathrm{MPa}$, elongation to failure $\sim 27 \%$ ). After the parts were created, they were cleaned using a Branson 3200 Ultrasonic cleaner to remove any uncured resin. The layered building process inherent to stereolithography produces a very rough surface finish on the prototypes, which increases the engagement force and impedes motion due to excessive friction. To improve this finish, liquid resin was hand applied to the lead surface of each prong and post. The prototype was then cured for 15 minutes in a 3D-Systems Post-Cure Apparatus (PCA). A second coat of liquid resin was applied to the lead surfaces and the prototype was cured for 60 additional minutes (Figure 10).

\section{Experimental Procedure}

Similar laboratory setups and procedures were used for the different operational scenario experiments. To examine the kinematics and stiffness of the mechanism, an experimental setup capable of measuring the load on the actuation triangle and prong tip deflection in response to applied actuation triangle displacements was constructed. Displacements were applied to the actuation triangle using a Newport UMR 12.40 single-
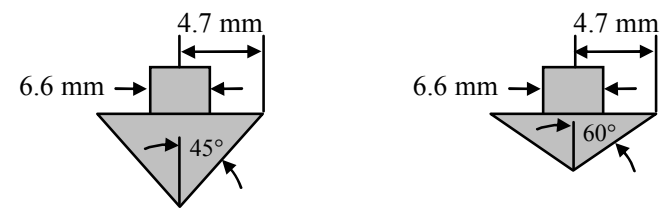

Figure 9: Guest Surface Post Dimensions. Two post designs, differing only in the conical half-angle, were used to examine the engagement behavior. A $4.7 \mathrm{~mm}$ maximum post radius, $R_{p o}$, was used for both samples while the lead surface conical half-angles were $45^{\circ}$ and $60^{\circ}$. axis load bearing stage (Figure 11). By affixing a $25 \mathrm{lb}$ Cooper Industries LPM 530 force transducer between the stage and the actuation triangle, the load on the actuation triangle could be measured simultaneously with the applied displacement. Because the prong's angle relative to the host surface changes significantly as it deflects ( $\sim 10 \mathrm{deg}$ for the current design), available laser and fiber optic displacement sensors could not be employed to measure its deflection. Alternatively, the prong's position in space was measured using an aluminum probe mounted to a Newport 462-XYZ-M three-axis precision stage via a $5 \mathrm{~g}$ Cooper Industries LPM 620 force transducer. The aluminum probe was positioned using the three-axis stage until contact was made at the center of the prong connection topology allowing its position to be measured in three dimensions. To insure that the aluminum probe did not significantly alter the prong position, the force imparted by the probe was minimized using the sensitive $5 \mathrm{~g}$ force transducer.

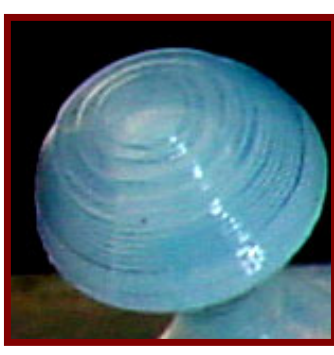

a) Unfinished Rough Surface

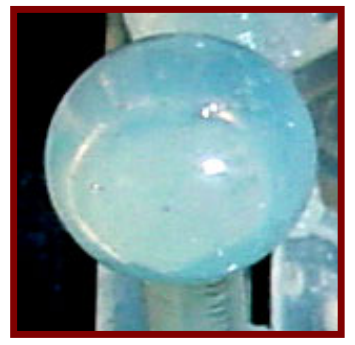

b) Finished Smooth Surface
Figure 10: Surface Finish Improvement. Two coats of uncured Somos 8110 resin were hand applied to the lead surfaces of the connection elements to minimize friction during engagement and motion generation. The finished surfaces were then fully cured in a 3D-Systems PCA. 


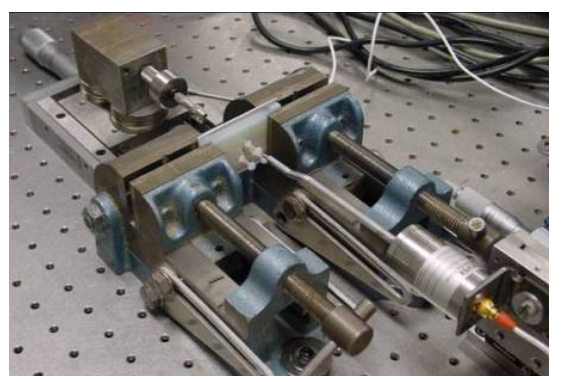

a) Single-Cell Test

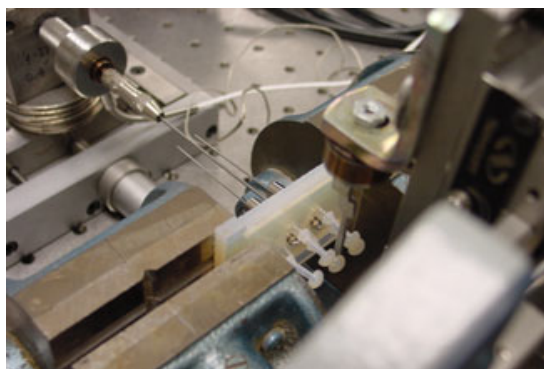

b) Three-Cell Test

Figure 11: Experimental Test Setup. For both the single and three-cell tests displacements were imparted on the actuation triangles using a precision Newport single-axis stage. a) The single-cell free deflections of the prongs were measured using an aluminum probe attached to a three-axis Newport precision sage. This probe was attached to the stage through a $5 \mathrm{~g}$ force probe to insure that the prong position was measured accurately without loading the prongs significantly. For engagement and retention testing the aluminum probe was replaced with a single-post guest surface. b) For the three-cell samples the force at the tip of the Prong $B_{1}$ (Figure 2) was measured using an aluminum probe. The probe was mounted via a $10 \mathrm{lb}$ Cooper Instruments force probe to a three-axis Newport precision stage. The probe was positioned using the three-axis stage to map out the force-deflection curve for the three-cell samples.

The setup was modified slightly to permit the examination of the single-cell engagement and retention behaviors by removing the aluminum probe and replacing it with a single-post guest surface. By mounting the guest surface to the Newport 462-XYZ-M via a $10 \mathrm{lb}$ Cooper Industries LPM 530 force transducer, both the position and force imparted in the guest surface could be measured simultaneously. For the engagement and retention tests, the actuation triangles were separated from the single-axis UMR 12.40 stage to permit their free deflection in response to the guest surface motions.

\section{SINGLE-CELL BEHAVIOR}

The free deflection kinematics of the mechanism (Figures 12a) was examined to verify the accuracy of the model in the absence of cell interactions and frictional effects. As observed in Figure 14a, the model captured the prong deflection extremely well with an average error of $1.7 \%$ between both cases. More importantly it was demonstrated that the design could be tailored to the requirements of the intended actuation source. Design 1 required approximately $0.64 \mathrm{~mm}$ of actuation deflection and $6.1 \mathrm{~N}$ of force to fully deflect the prongs thus representing a low stroke, moderate force actuator requirement. In contrast design 2 requires a moderate stroke, low force actuation system with $1.6 \mathrm{~mm}$ of required deflection and $4.5 \mathrm{~N}$ of force. It is important to note that the two designs require differing degrees of prong deflection to reach the fully deflected state where the prongs are all touching each other. Since the prongs are typically deflected as far as possible during operation this state was used as the comparison point between the designs. Also of note is that the tip deflection of the prong was linear with respect to the input displacement of the actuation triangle. This is not often the case with compliant mechanisms and can be highly beneficial when developing a control system for the design.

The stiffness at the actuation triangle was measured to determine if the material was modeled correctly and

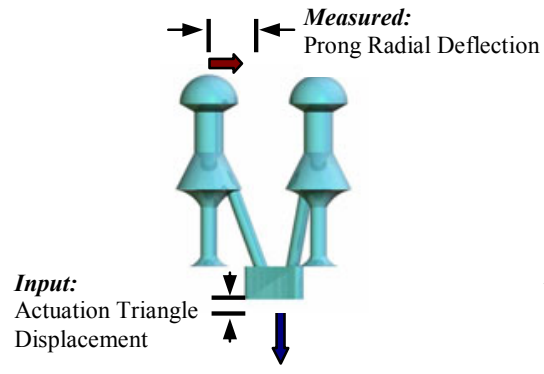

a) Kinematics

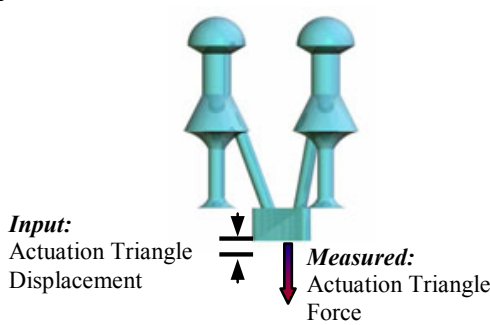

b) Stiffness at the Actuation Triangle

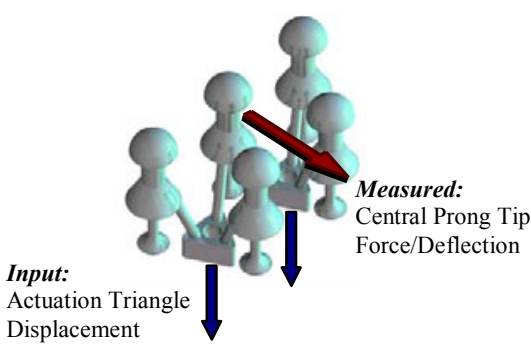

c) Positioning Force

Figure 12 Experimental Input/Output Parameters. a) To examine the kinematics of the mechanism, the relationship between the input displacement at the actuation triangle and the resulting displacement of the prong tips was experimentally measured. b) The models ability to accurately account for the stiffness of the prong column and suspension lines was examined by measuring the force required to enact deflection of the actuation triangle. c) The potential positioning force was examined using a three cell host surface verifying the modelís ability to capture cell interactions. 


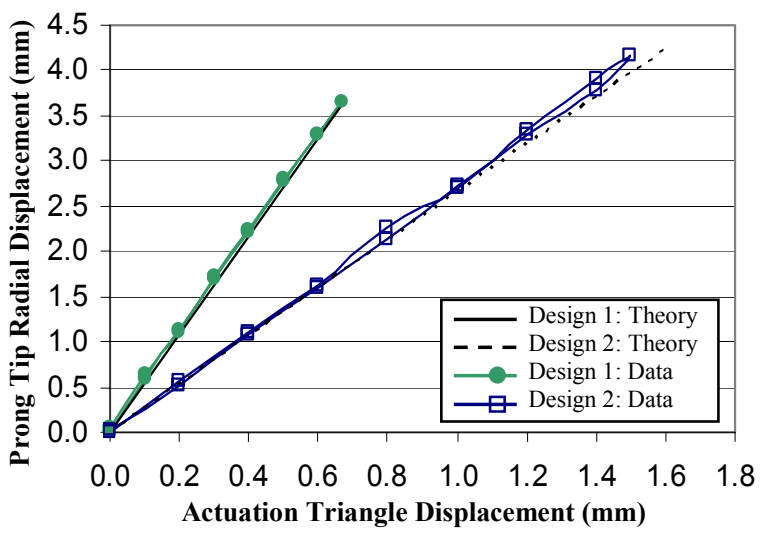

a) Kinematics

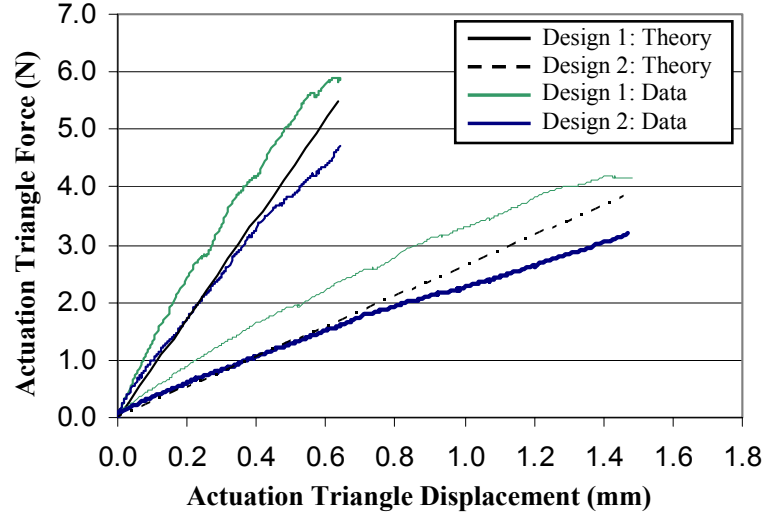

b) Stiffness at Actuation Triangle

Figure 13: Single Cell Kinematics and Stiffness at the Actuation Triangle. a) Prong tip radial deflection in response to displacements imparted to the actuation triangle was measured to verify the accuracy of the modelís kinematics predictions without considering the build material modulus. A linear prong response for both designs was measured with design 1 requiring $0.6 \mathrm{~mm}$ of actuation stroke to generate $3.6 \mathrm{~mm}$ of prong deflection and design 2 requiring $1.5 \mathrm{~mm}$ of actuation stroke to generate $4.2 \mathrm{~mm}$ of prong deflection. The model accurately captured the prong deflection with an average error of $1.7 \%$ from the experimentally measured response. b) The force-deflection relationship at the actuation triangle was measured to determine if the model accurately captured the material behavior of the build material. Nonlinearity in the force-measurements was observed as a result of plastic deformations of the prong columns and the suspension lines. With displacement applied rapidly over 2 seconds, the response was linear, if applied over 60 seconds, over $23 \%$ depression of the force was observed. In the case of applying the deflection over 60 seconds, $18 \%$ error in the force measurements was obtained.

particularly if it exhibited any nonlinearities (Figure 12b). Because the actuators will be attached to the actuation triangle, an accurate estimate of the stiffness at this location is important for stiffness matching the actuator for maximum energy transmission. The forcedefection relationship for Design 1 (Figure 13b) demonstrates the plastic deformations occurring in the prong columns and suspensions as the force required to maintain a given prong deflection decreased with time. When the displacement was applied rapidly over 2 seconds, the response was linear but when applied slowly over 60 seconds, a $23 \%$ depression in the force at the actuation triangle was observed. This plastic deformation resulted in an average error of $18 \%$ from theory (Equation 12). After the tests, residual displacements of the actuation triangle were up to $13 \%$ of the applied deflections indicating elevated stress levels leading to plastic deformation during the testing.

\section{POSITIONING FoRCE}

The interaction between cells was introduced by validating the three-cell model (Equation 28). The force-deflection response at the tip of the shared prong $B_{1}$ was measured in response to an applied $0.57 \mathrm{~mm}$ displacement of the actuation triangles (Figures $11 \mathrm{~b}$ and 12c). This displacement results in the prongs fully deflecting (contacting each other) under free deflection conditions. Results from this test bound the positioning force available for motion generation as it eliminates friction and resistive effects between the guest surface post and host surface prongs. Testing the more complex three-cell test bed revealed excellent correlation between the model and observed behavior (Figure 14). Over two trials with the same test bed, an average blocked force of $0.71 \mathrm{~N}$ and an average free deflection of $2.77 \mathrm{~mm}$ were observed representing a $4.7 \%$ error in blocked force and $5.9 \%$ error in free deflection. On average for the entire force-deflection

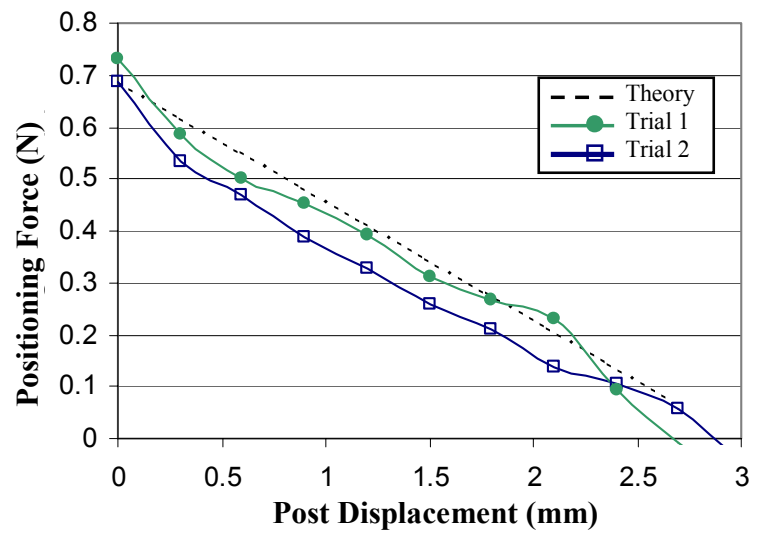

Figure 14: Positioning Force. The potential positioning forces produced during motion generation were examined using a threecell host surface. Since the test did not include a guest surface post, no frictional losses were present thereby representing the upper bound on the potential positioning forces. Trial 1 produced a blocked force of $0.73 \mathrm{~N}$ and a free deflection of $2.7 \mathrm{~mm}$ while trial 2 produced $0.69 \mathrm{~N}$ and $2.9 \mathrm{~mm}$. On average the model was accurate to within $6.1 \%$ across the entire force-deflection curve. During the testing, plastic deformation of the prongs was observed near the blocked force location making it difficult to discern the blocked force location and magnitude. This error in testing led to the approximately $7 \%$ shift in the force-deflection curves between trials. 
curve, the model was accurate to within $6.1 \%$. Much of this error stems from the difficulty in locating the blocked force position due to plastic deformation of the prongs between tests and was not representative of changes in mechanism performance between trials. This is the reason that the two trials are shifted apart by approximately $7 \%$. The three-cell testing demonstrated that useful forces of up to $0.71 \mathrm{~N}$ of positioning force could be applied to a guest surface post by a single cell with the present configuration in comparison to other micro-scaled active surfaces that only provide on the order of millinewtons of force. Because the force increases linearly with the number of posts on the guest, translational and rotational forces of several newtons are readily attainable.

\section{ENGAGEMENT}

Frictional effects were examined by the introduction of the post in a single-cell host surface. Single-cell engagement testing demonstrated that successful attachment of guest and host surfaces could be accomplished with minimal force $(<0.58 \mathrm{~N})$ even in the presence of friction (Figure 15). The general shape of the engagement behavior was captured well with a linear initial stage up to the transition point, and a nonlinear response after the transition point with the slope of the curve decreasing until engagement is complete. Generally the model was accurate up to about $1.6 \mathrm{~mm}$ of post displacement for the $60^{\circ}$ halfangle guest surface with an average error of $9.8 \%$ from the $\mu_{\mathrm{E}}=0.1$ theory and $15.6 \%$ from the $\mu_{\mathrm{E}}=0.2$ theory. After $1.6 \mathrm{~mm}$ the force dropped off due to deviations in the prong shape from the idealized hemispherical shape.

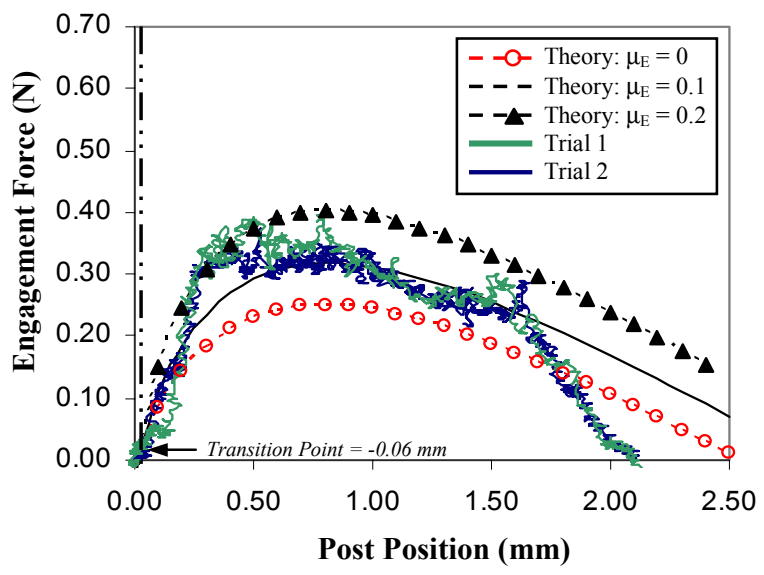

a) $60^{\circ}$ Guest Surface
Over two trials, the $60^{\circ}$ guest surfaces required an average maximum force of $0.39 \mathrm{~N}$ to engage compared to a predicted maximum of $0.32 \mathrm{~N}$ for an estimated $\mu_{\mathrm{E}}=0.1$ and $0.40 \mathrm{~N}$ for $\mu_{\mathrm{E}}=0.2$. Inconsistent friction levels were evidenced by the jagged force measurements and the overall trend in the data where it tracked the $\mu_{\mathrm{E}}=0.2$ theory well up to $0.35 \mathrm{~mm}$ of post displacement before dropping down to the $\mu_{\mathrm{E}}=0.2$ theory line.

Testing of the $45^{\circ}$ half-angle guest surface yielded interesting results that highlighted the sensitivity of the design to manufacturing defects. Up to a post displacement of $0.25 \mathrm{~mm}$ the response tracked theory well with an average error of only $6.7 \%$ for an estimated $\mu_{\mathrm{E}}=0.1$ and $7.6 \%$ for $\mu_{\mathrm{E}}=0.2$. At $0.25 \mathrm{~mm}$ of post displacement the prong and post began to stick together instead of sliding smoothly across each other and the force rose sharply requiring a maximum force of $0.58 \mathrm{~N}$ to engage compared to a predicted maximums of $0.32 \mathrm{~N}$ and $0.40 \mathrm{~N}$ for estimated friction levels of $\mu_{\mathrm{E}}=0.1$ and $\mu_{\mathrm{E}}=0.2$ respectively. The deviations from the predicted behavior are the result of three factors stemming from the shortcomings of the stereolithography fabrication method employed: the prong is not perfectly hemispherical, the post is not perfectly conical, and the friction levels are not constant across the surfaces.

\section{RETENTION}

Frictional variances also played an important role in the observed retention behavior. The hand coating process employed on the leading surface was not applied to the

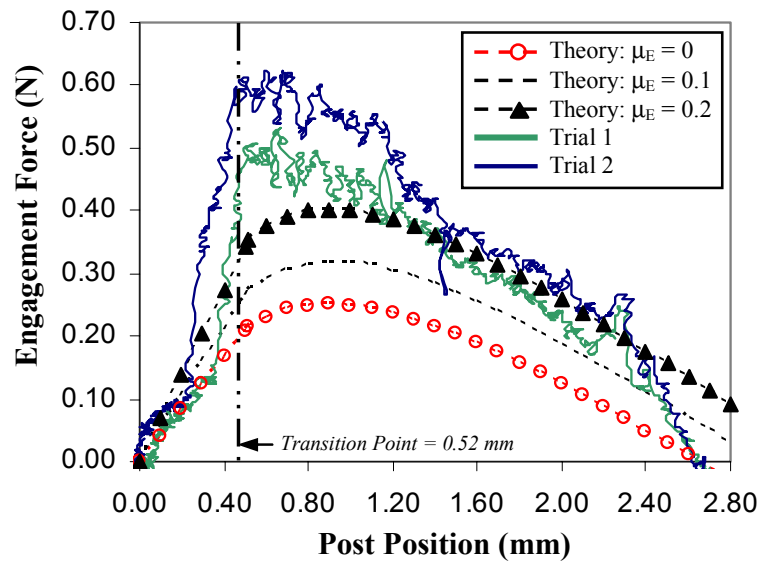

b) $45^{\circ}$ Guest Surface

Figure 15: Engagement Force. The relationship between the engagement force and the imparted guest surface post displacement was examined and compared to model predictions. Two guest surfaces were fabricated with identical $4.7 \mathrm{~mm}$ radius conical lead surface but varied cone half-angles, $60^{\circ}$ and $45^{\circ}$. The $60^{\circ}$ guest surface required an average force of $0.39 \mathrm{~N}$ to engage compared to the predicted force of $0.32 \mathrm{~N}$ and $0.40 \mathrm{~N}$ for estimated friction levels of $\mu_{\mathrm{E}}=0.1$ and $\mu_{\mathrm{E}}=0.2$ respectively. The $45^{\circ}$ guest surface response was not predicted accurately due to excess levels of friction and unsmooth guest and host surfaces. This caused the engagement response to increase sharply after about $0.25 \mathrm{~mm}$ of post displacement. Up to this point the model predicted the response accurately. An average force of $0.58 \mathrm{~N}$ was required to engage the $45^{\circ}$ guest surface well above the predicted force of $0.32 \mathrm{~N}$ for $\mu_{\mathrm{E}}=0.1$ and $0.40 \mathrm{~N}$ for $\mu_{\mathrm{E}}=0.2$. 
trailing edge surface. For all samples, the surfaces were sanded with 200 and 400 grit sand paper until they felt smooth to the touch but clearly the ridges were not completely eliminated or new ridges were introduced in the finishing process. However it was difficult to fully smooth the surface without affecting the shape. Doing so resulted in post and prong trailing surfaces that were not mutually parallel.

Experimental testing of the retention behavior clearly indicates that the trailing surface were not smooth as the force-deflection response was quite jagged. Even with the jagged response the general trend is still clear throughout the separation process (Figure 16). Two test runs were performed resulting in an average maximum recorded retention force of $2.1 \mathrm{~N}$ compared to a predicted value of $2.2 \mathrm{~N}$ for the frictionless case and $2.85 \mathrm{~N}$ for a low friction case of $\mu_{\mathrm{R}}=0.05$. The experimentally observed retention force was up to 5.4 times greater than the engagement force for identical surfaces. This feature of the Active Velcro technology allows the engagement forces to be kept small to insure a successful attachment while simultaneously creating a substantial retention force to prevent undesired guest surface separation.

As evident in the theory lines of Figure 16 the retention behavior is highly sensitive to friction. Furthermore the evenly spaced "teeth" in the measured response highlight the minute ridges present on the post and prong trailing surfaces from the stereolithography process. Prior to the sanding, the ridges were sufficient to prevent separation of the guest surface post without damaging the prongs, hence creating a significant retention force. Preliminary testing with unfinished surfaces generated retention forces in excess of the 25 lb Cooper force probe's limitations.

\section{CONCLUSIONS}

Active Velcro is a unique distributed actuation surface that provides controlled retention of the guest surface, unlike the present active surface technologies. This paper focused on the mechanism that provides this connection. A quasi-static force-deflection model based upon the Matrix Displacement Approach was presented. This is a generalized model and specific cases were highlighted for the main operating scenarios: engagement, retention and positioning force. While the primary model development was based upon a single-cell, the methodology to expand the model for multiple cells was provided with a three-cell example given.

Several experiments were conducted to progressively examine the accuracy of the derived models and the behavior of the Active Velcro technology. In freedeflection experiments, the analytical model captured the kinematics of single-cell host surfaces with an

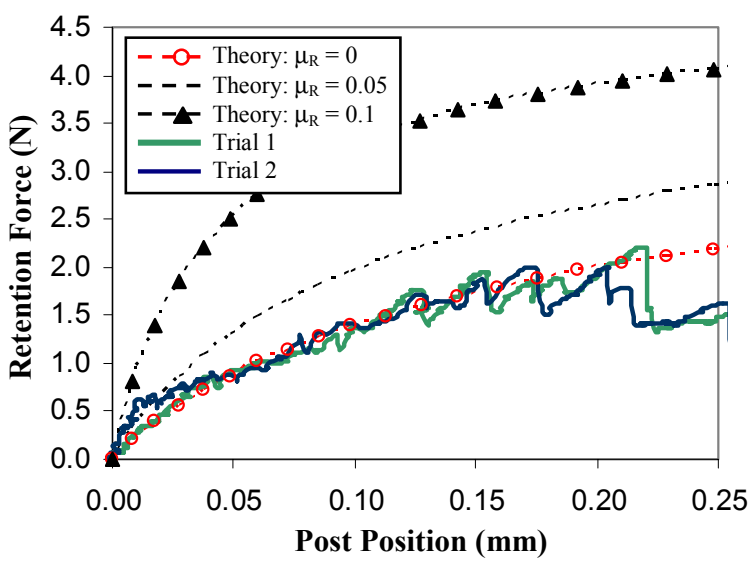

Figure 16: Retention Force. The relationship between the retention force and the imparted guest surface post displacement was examined and compared to model predictions. Three trial runs were performed using the same host and guest surfaces resulting in an average maximum recorded retention force of $2.1 \mathrm{~N}$ compared to a predicted value of $2.2 \mathrm{~N}$ for the frictionless case and $2.85 \mathrm{~N}$ for a low friction case of $\mu_{R}=0.05$. Retention was highly sensitive to friction levels particularly at the onset of surface separation. For friction coefficients above approximately $\mu_{R}=0.13$, the theory predicts that the surface will not separate.

average error of only $1.7 \%$. Two different test samples were employed, demonstrating the ability to tailor the input requirements of the mechanism to desired actuator specifications. The observed prong displacement was linear with respect to the actuation inputs, which is uncommon for high displacement, compliant mechanisms. The large displacements however did lead to as much as $23 \%$ plastic deformation.

It was observed in both the engagement and retention experiments that the model captures the general shape of the response curve well. As designed, engagement occurs at a low force $(<0.58 \mathrm{~N})$ even for non-ideal surfaces and the retention forces are significantly higher than the engagement forces, $3.7-5.4$ times higher for the samples tested. Despite the post-processing methods created, irregularities in friction levels were observed in both the engagement and retention tests leading to errors on average of $19 \%$. These frictional variances stemmed from the layered stereolithography fabrication method employed to build the test samples and was exasperated by the relatively thick minimum layer size of $150 \mu \mathrm{m}$ for the SLA-250/40 utilized in this research. In addition, the shapes of the heads of the post and prong were not ideal. In light of the manufacturing difficulties and unknown frictional surfaces, the model captured the overall performance well, typically within $9.8 \%$ for surfaces resembling the assumed post and prong connection topology shapes.

Multi-cell tests were performed to validate the model in the presence of cell interactions. The model was 
capable of capturing the positioning force with fewer than $6.1 \%$ error. While the model presented does not incorporate the frictional interaction with the post, it does bound the maximum force that the host can impart to position the guest surface. In the sample tested, this was $0.71 \mathrm{~N}$ which is one to two orders of magnitude greater than those of current MEMS based systems. If multiple posts are used, several newtons of force are possible making it a viable technology for complex motion generation in the low gravity environment of space, the turbulent underwater environment, or aboard moving ground, sea or air vehicles.

\section{ACKNOWLEDGMENTS}

This paper is based upon research supported the Defense Advanced Research Projects Agency and the Air Force Research Laboratory under contract number F29601-98-D-0210.

\section{References}

${ }^{1}$ Suh, J.W., Darling, R.B., Bohringer, K.F., Donald, B.R., Baltes, H., Kovacs, G.T.A., 1999, "CMOS Integrated Ciliary Actuator Array as a General-Purpose Micromanipulation Tool for Small Objects," Journal of Microelectromechanical Systems, Dec, Vol. 8, No. 4, pp $483-496$.

${ }^{2}$ Ebefors, T., Mattsson, J.U., Kalvesten, E., Stemme, G., 1999, "A Robust Micro Conveyer Realized by Arrayed Polyimide Joint Actuators," in Proc. of the IEEE International Conference on Micro Electro Mechanical Systems, Jan. 17-21, pp 576-581.

${ }^{3}$ Ataka, M., Omodaka, A., Takeshima, N., Fujita, H., 1993, "Fabrication and Operation of Polyimide Bimorph Actuators for a Ciliary Motion System," Journal of Microelectromechanical Systems, Dec, Vol. 2, No. 4, pp 146 - 150.

${ }^{4}$ Langlet, P., Collard, D., Akiyama, T., Fujita, H., 1997, "A Quantitative Analysis of Scratch Drive Actuation for Integrated X/Y Motion System," International Conference on Solid State Sensors and Actuators, June 16-19, Vol. 2, pp $773-776$.

${ }^{5}$ Iizuka, T., Fujita, H., 1997, "Precise Positioning of a Micro Conveyor Based on Superconducting Magnetic Levitation," in Proc. of the IEEE International Symposium on Micromechatronics and Human Science, Oct. 5-8, pp $131-135$.

${ }^{6}$ Nakazawa, H., Watanabe, Y., Morita, O., Edo, M., Yonezawa, E., 1997, “The Two-Dimensional Micro Conveyer: Principles and Fabrication Process of the Actuator," International Conference on Solid State Sensors and Actuators, June 16-19, Vol. 1, pp 33 - 36.

${ }^{7}$ Bohringer, K.F., Donald, B.R., Mihailovich, R., MacDonald, N.C., 1994, "A Theory of Manipulation and Control for Microfabricated Actuator Arrays," in Proc. of the IEEE Workshop on Micro Electro Mechanical Systems, Jan. 25-28, pp $102-107$.

${ }^{8}$ Bohringer, K.F., Donald, B.R., MacDonald, N.C., 1999, "Programmable Force Fields for Distributed Manipulation, with Applications to MEMS Actuator Arrays and Vibratory Parts Feeders," International Journal of Robotics Research, Feb., Vol. 18, No. 2, p $168-200$.

${ }^{9}$ Konishi, S., Fujita, H., 1994, “A Conveyance System Using Air Flow Based on the Concept of Distributed Micro Motion Systems," Journal of Microelectromechanical Systems, June, Vol. 3, No. 2, pp $54-58$.

${ }^{10}$ Luntz, J.E., Messner, W., and Choset, H., 2000, "Closed-Loop Operation of Actuator Arrays," in Proc. of the IEEE Conference on Robotics and Automation, San Francisco, CA, April 24-28, Vol. 4, pp 3666 3672 .

${ }^{11}$ Tadokoro, S., Fuji, S., Fushimi, M., Kanno, R., Kimura, T., Takamori, T., Oguro, K., 1998, "Development of a Distributed Actuation Device Consisting of Soft Gel Actuator Elements," in Proc. of the IEEE Conference on Robotics and Automation, Leuven, Belgium, May 16-20, Vol. 3, pp 2155 - 2160.

${ }^{12}$ Clement, J.W. and Brei, D.E., 2001, "Proof-ofConcept Investigation of Active Velcro for Smart Attachment Mechanisms," in proceedings of the 42nd AIAA/ASME/ASCE/AHS/ASC Structures, Structural Dynamics, and Materials Conference, Seattle, WA.

${ }^{13}$ Pestel, E.C. and Leckie, F.A., 1963, "Matrix Methods in Elastomechanics," McGraw Hill Publishing Co., Inc., New York. 